\title{
User Interface Design of Mobile Photo Editors
}

\author{
Irma Rochmawati \\ Departemen Desain Komunikasi Visual \\ Universitas Komputer Indonesia \\ Bandung, Indonesia \\ irma@email.unikom.ac.id
}

\begin{abstract}
Capture many events with the camera have become the need of everyone. Advances in technology bring the smart phone is supported with a camera in it. The mobile application supports the interest of people in photography that provides features like image editing. Photo-editing apps are in great demand to produce the perfect picture. This allows designers to regularly engage in this kind of updates. There are many mobile applications available for photo editing such as Snapseed, VSCO, and Adobe Photoshop Express that can be downloaded for free via smart phone. The goal of interface design is to define a set of interface objects and actions, but how the interface of mobile applications can be understood by users, so that people can easily produce the perfect picture. In this research, visual descriptive method was used in testing photo editor mobile applications based on Android and iOS a platform. Well-designed apps can be successfully implemented if supported with a powerful interface. An effective user interface for photo-editing services helps non-designers create beautiful photos with no significant effort. The designer's job is to organize and present all the features in that way so that everyone can do photo editing effectively and intuitively. The result is mobile photo editors can be understood by the user if presented with a good user interface.
\end{abstract}

Keywords-design, mobile application, photo editors, user interface

\section{INTRODUCTION}

Mobile applications support people's interest in photography providing various features such as image editing. Today photo editing products are in high demand the reason why designers are regularly involved in projects of user interface of mobile apps. User Interface (UI) is what interacts with the user as part of an experience [1]. User interface is the connection between users and experience, first impressions, and lasting impression. A good user interface design must maintain a perfect balance between charming aesthetics and interactivity without requiring more effort [1].

At this time, there is a lot of research on user interface design. But not many have discussed the interface of a photo editor application. User interfaces come in a numerous different forms, some good but many more bad. The look, feel and general usability of a user interface is extremely important, especially on a mobile application. A badly designed user interface typically leaves a user with a poor lasting impression of the company with which the interface is associated [2]. Stone et al. (2005) indicate that a good user interface design promotes easy, natural, and engaging interaction between the user and the system, making the user forget that s/he is using a computer [3]. However, there are some problems. Not everyone can use the editing feature in an application, especially non-professional users. User interface is not only about color and shape, but about presenting the right tools to the user to be able to achieve its goals. In addition, the user interface is more than just buttons, menus, and forms that must be filled by the user [1]. The goal of interface design is to define a set of interface objects and actions (and their screen representations) that enable a user to perform all defined tasks in a manner that meets every usability goal defined for the system [4]. As the iterative design process continues, a user interface tool kit may be used to complete the construction of the interface [4].

My previous research was comparing the interface design of the three photo editor applications, then the goal of this research is to find out how good the interface of a photo editor of mobile app can help people understand the use of photo editor features. This work investigates how to construct a workflow for creating high quality image editing on mobile application.

Android's default user interface is mainly based on direct manipulation, using touch inputs that loosely correspond to real-world actions, like swiping, tapping, pinching, and reverse pinching to manipulate on-screen objects, along with a virtual keyboard [5]. Other than that, iOS is a mobile operating system created and developed by Apple Inc. It is the operating system that presently powers many of the company's mobile devices, including the iPhone, iPad, and iPod Touch. It is the second most popular mobile operating system globally after Android. The iOS user interface is based upon direct manipulation, using multi-touch gestures. Interface control elements consist of sliders, switches, and buttons. Interaction with the OS includes gestures such as swipe, tap, pinch, and reverse pinch, all of which have specific definitions within the context of the iOS operating system and its multi-touch interface. Apple has been significantly praised for incorporating thorough accessibility functions into iOS, enabling users with vision and hearing disabilities to properly use its products.

\section{METHOD}

This research was conducted by testing on mobile devices. Some photo editor mobile applications that were studied i.e. Snapseed, VSCO, and Adobe Photoshop Express. To support this research, visual descriptive method was used in testing photo editor mobile applications based on Android and iOS platform. This method is useful because it can find out the quality of the user interface design of the photo editor 
mobile applications used by non-professional users. There are three stages to achieve the objectives in this study.

\section{A. Selecting a Device}

In this research used device which is a smartphone based on Android or iOS platform. Android is a mobile operating system developed by Google, designed primarily for touchscreen mobile devices such as smartphones and tablets.

\section{B. Installing the Apps}

In previous study, three photo editor applications were chosen among popular Android and iOS-based smartphone users, that is Snapseed, VSCO, and Adobe Photoshop Express. All three are installed on the device. Then a sample test was conducted which in this study Snapseed was chosen as the best one.

VSCO is more than just photo editing, it offers a community for photographers to connect and create. VSCO X is a new paid version of VSCO Camera that's costly without adding enough value to justify the cost. The company plans to turn it into an educational platform for photography and imaging, and when they have more lessons online. This is the reason why this application was eliminated.

Adobe Photoshop Express reflects its heritage in highquality photo editing. However, on the mobile side of things, Snapseed offers more versatility for the consumer, and it is far more powerful and flexible for serious photographers.

\section{Trial and Error}

Most of mobile cameras these days have Auto Focus. Probably people not know this part. When you tap the screen on camera view, camera will focus on the nearest object. This is how most cameras work. Most cameras have this trial and error sort of feature built into it to help you select the right object to focus on. Other than that, some applications don't save images automatically if we don't export them to the storage gallery.

\section{RESULTS}

A good interface can be understood by the user. after being observed, it can be investigated how to construct a workflow for creating high quality image editing on mobile application. The way is quite simple, users just open a photo, then edits and save.

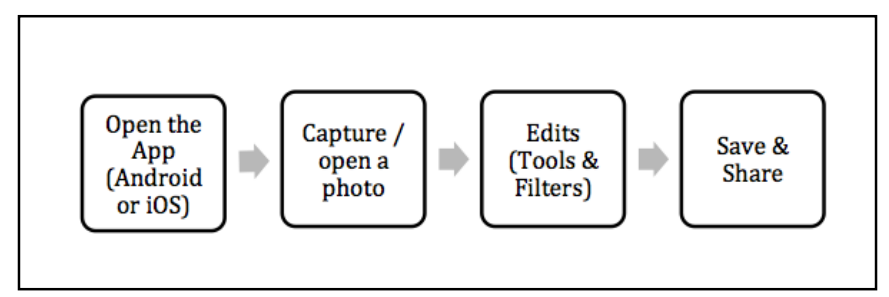

Fig. 1. Typical photo editing process.

\section{A. Versatility of Camera Module}

Mobile photography looks really simple on the surface. But when you start digging deeper, it's really not that simple. The mobile camera has so many hidden features and tricks that you probably don't know about.

Snapseed camera has a simple look of interface (Fig. 2). VSCO also offers a built in camera with advanced controls, allowing you to capture the perfect shot, edit and save, all without leaving the app.

A digital camera measures red, green and blue light separately, then analyzes these measurements to create colors. However, there are a lot more colors that a camera can measure than are visible by the human eye. For example, JPEG files are images made up of actual colors that the eye can see. Extra color data gets clipped and simplified, like the brightest and darkest parts of the image appearing solid white or solid black.

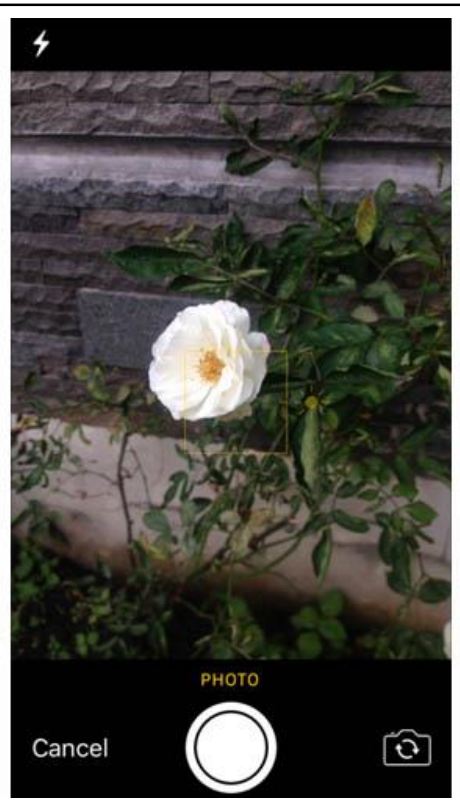

Fig. 2. Camera view in Snapseed.

\section{B. Quality and Accessibility of the Interface}

In addition to the basic editing features making photo editing easier than ever. Many photo editing applications make this process cumbersome, but Snapseed keeps it simple and offers many options that can be easily adjusted. Of course, you can change the lighting and add filters, but the ability to refocus the image and change the style of the film makes their toolbox unique. And finally, based on the saving, re-editing capabilities you've built in the past, make this app be everyone favorite photo editor.

\section{Number and Quality of Features}

Snapseed is professional level photo editing with an intuitive interface that's simple to use but doesn't compromise on functionality. Snapseed offers a versatile photo editing app for mobile. When you have captured an image, the interface offer many looks of filters (Fig. 3). Such as Smooth, Faded Glow, Fine Art, Silhouette, and many more.

At center point of editing, Snapseed has tools for best adjustments. There are 28 features in total (Fig. 4). Besides the standard editing functions like Rotate, Crop, and White Balance, Snapseed is also loaded with extra powerfull features like the Brush, Tune Image, Perspective, and Text. For filters you can try Grunge, Grainy Film, Vintage, and Retrolux. 


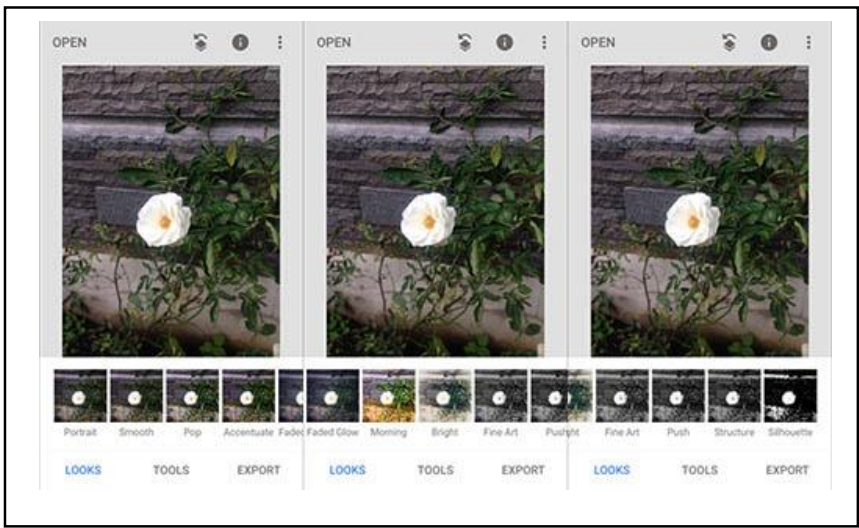

Fig. 3. Feature LOOKS in Snapseed.

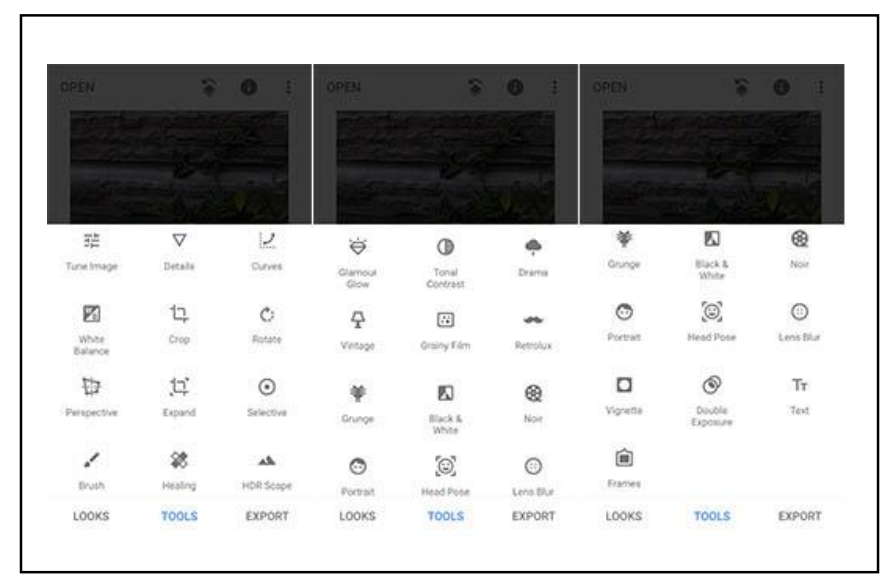

Fig. 4. Feature TOOLS in Snapseed.

\section{Save and Sharing}

After editing a photo on mobile application, users are given the easiness of saving the image editing results with storage features. Snapseed also offers sharing edits results to another application such as Instagram, Facebook or Twitter. The interface of this feature is equipped with icons and text and clearly made the difference between Save and Export. Snapseed edits saved with Save or Save a copy may not appear on images when accessed through certain third party applications. To ensure that Snapseed edits are viewable by all third party applications, users need to select Export.

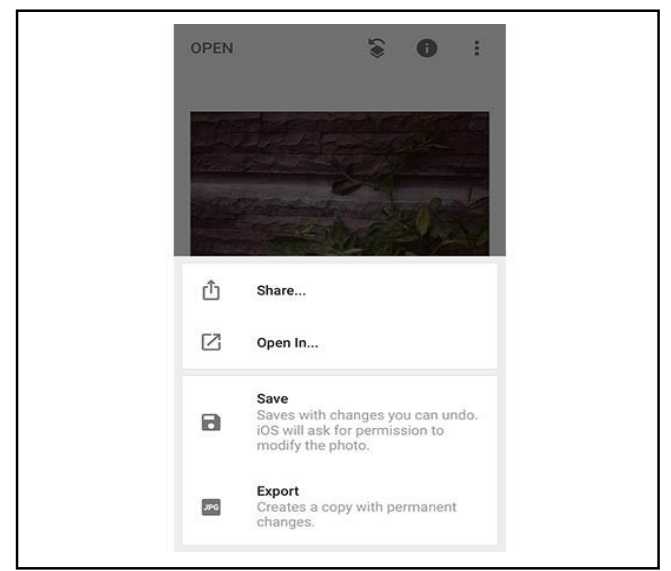

Fig. 5. Save and share interface in Snapseed.

\section{DISCUSSION}

Well-designed apps can be successfully implemented if supported with a powerful interface. An effective user interface for photo editing services helps non-designers create beautiful photos with no significant effort.

Snapseed is a photo-editing application produced by Nik Software, now owned by Google. Snapseed supported for Android and iOS platform that enables users to enhance photos and apply digital filters. Snapseed was rated as one of the Top 100 Best Android Apps of 2018 by PC Magazine [6]. Snapseed is a comprehensive photo editing application, isn't for the casual user, but for serious photographers who want or need to spend time creating the best possible image while on the go [7]. But how the interface of mobile applications can be understood by users, so that people can easily produce the perfect picture even they are not a serious photographers.

Snapseed uses gestures to make edits to photos. For instance, in the Tone Image tool, swipe up or down to select from among Brightness, Contrast, Saturation, Ambience, Highlights, Shadows and Warmth. Then swipe left or right to increase or decrease the intensity of that effect. While this is the kind of information that is well-documented in Snapseed's generally helpful tutorials, other key controls and gestures, such as pinching to resize the brushes, are not as clearly explained and are often discoverable only by talking with other users or by experimenting [8].

The biggest problem we had with Snapseed is the lack of an auto-save as edits. So if you accidentally tap the back button before you save, you will lose all your work. Sharing is an afterthought, because that activity isn't photocentric in the traditional sense. However, we found it quite easy to post images to Facebook and other apps installed on our devices [8].

Snapseed continues to add to its arsenal of photo tools. For the most part, they are quite useful and effective, such as the new Curves exposure dialog. However, I had mixed results with the new Expand tool, which enlarges a picture by copying image data to fill space on the new outer edges. The problem with the Expand tool is that it can't invent data it doesn't have. So it will work only on those photographs in which there's a certain uniformity along the edges, such as a field of grass or a clear blue sky.

\section{A. Essential Editing in Snapseed}

In editing a photo, requires a device that can provide various features. The essential editing in Snapseed can be done by the following steps.

1) Open an image: For editing a photo, follow this step by opening the gallery from device, or capture an image using camera within it. Here at the main screen, with selected photo open in its current state (Fig. 6).

2) Select a Tool: To start editing, tap the TOOLS tab. This shows all of Snapseed's editing tools. Scroll horizontally to view the options, then tap to select. I've chosen Tune Image, as it is a great place to start for basic edits like Brightness and Saturation. 


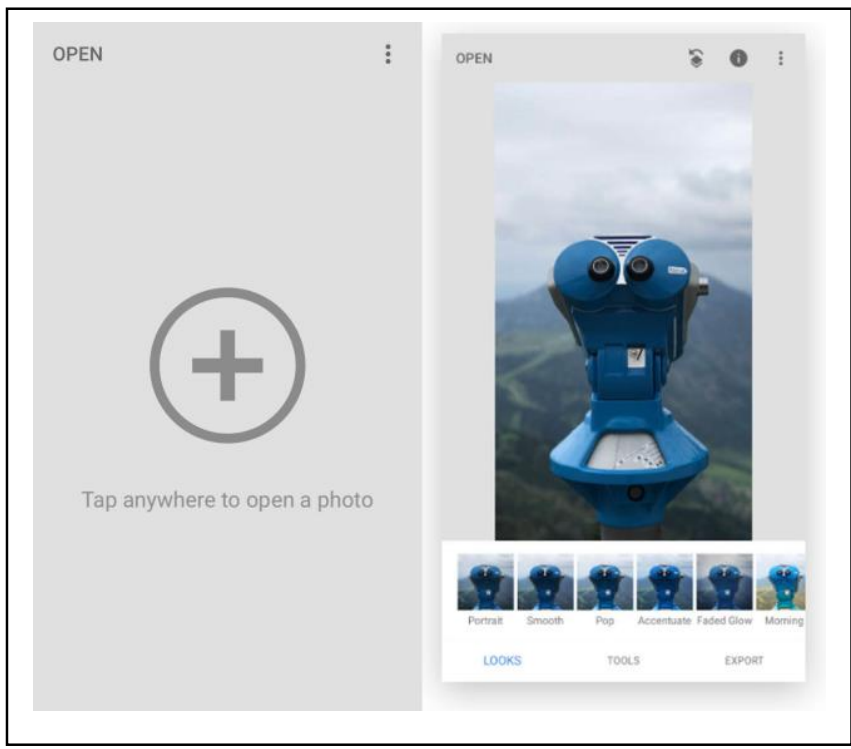

Fig. 6. Opening an image in Snapseed.

3) Swipe on the image to adjust: Most adjustments in Snapseed work this way: scroll vertically on the image to select a parameter, then swipe horizontally to change the value of the parameter. Here I've adjusted parameters including Brightness, Ambiance, Contrast, and Shadows. To apply the changes, tap on the checkmark. To cancel the changes, tap on the X. To add another tool, select one in the TOOLS list again.

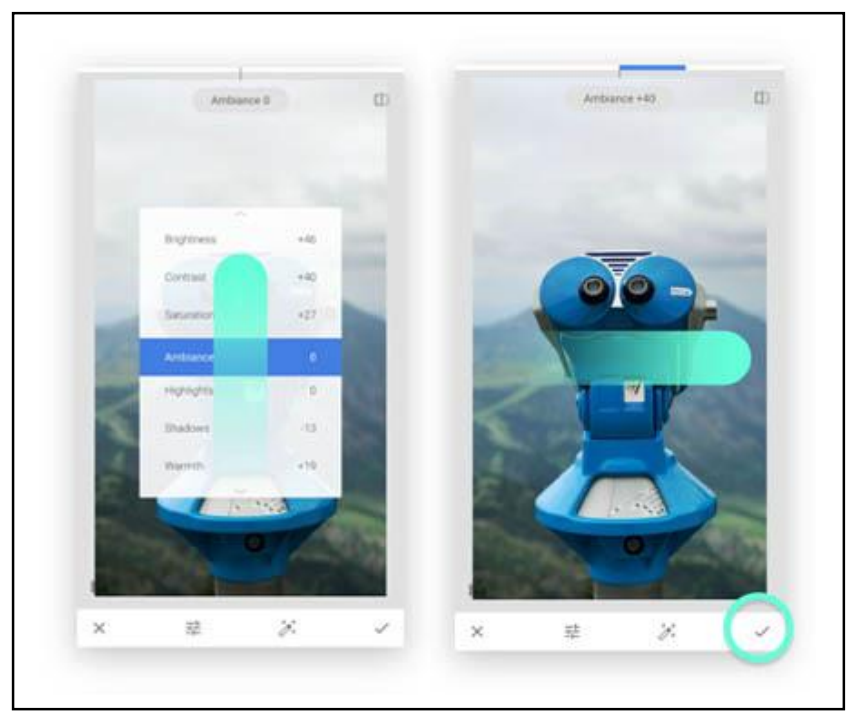

Fig. 7. Adjustment in Snapseed.

4) Start with a preset style: Many of the Snapseed Filters also come with pre-adjusted style that make good starting point for editing. In Grainy Film, for example, tap on the cards icon to open its list of options (Fig. 8). Scroll horizontally to view all the options, then tap to select. Experiment with the photo by adding more and more edits.

5) Save your image: To save the changes, tap the save icon, which is the left icon on the upper right corner of main screen.

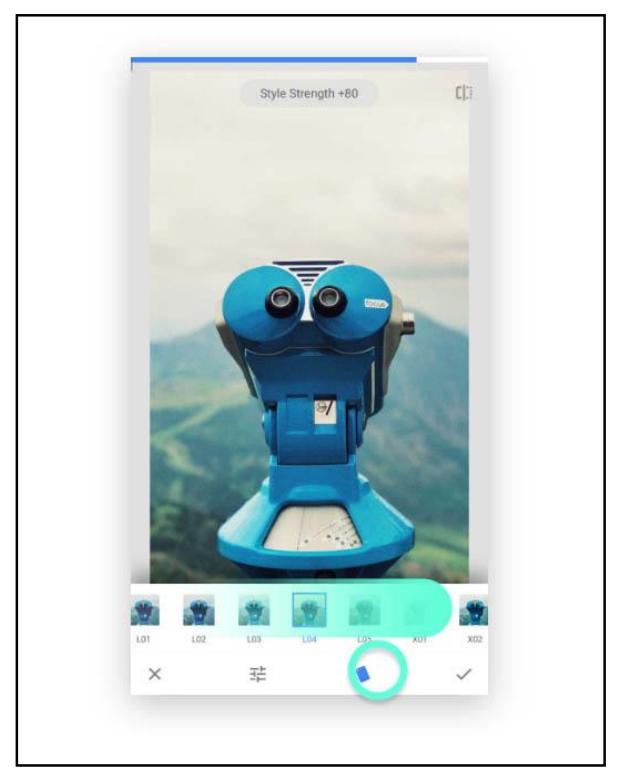

Fig. 8. Preset style in Snapseed.

The affordances and visibility make the discoverability happen. Letting the user to realise what is possible to do. To help the user to know how to use the controls there should be enough immediate feedback as well as constraints in place. Another term is natural mapping which means showing the relationship between the action and the controlled object in an understandable and natural way [2].

\section{CONCLUSION}

In editing a photo, requires a device that can provide various features. What really matters is not the way to get to know about user interface design, but the way an application can provide people to understand of user interface design. Moreover, people need to make it a chance to show by following some expert at photo editing and keep learning from those via website or any other channels.

\section{ACKNOWLEDGMENT}

This research was supported by Indonesia Computer University (UNIKOM) Bandung. I would like to thank our colleagues from Visual Communication Design who provided insight and expertise that greatly assisted the research. And also thank to reviewers for their insights in this paper.

\section{REFERENCES}

[1] C. Bank, J. Cao, "Web UI design best practices." Mountain View: UXPin, 2014.

[2] A. J. Stanford-Clark, "User interface design," New York: Patent, 2005.

[3] D. Stone, C. Jarrett, M. Woodroffe, S. Minocha, "User interface design and evaluation," Elsevier, 2005.

[4] S. Sridevi, "User Interface Design." International Journal of Computer Science and Information Technology Research ISSN 2348-120X (online) Vol.2, Issue 2, pp: (415-426), Month: April-June 2014. www.researchpublish.com, 2014.

[5] Billiondevices, http://www.billiondevices.com/

[6] D. A. Norman, "The design of everyday things," Basic Books, 2013. 
[7] M. Eddy, "The 100 Best Android Apps of 2018." pcmag.com. PC Magazine, July 7, 2018.

[8] S.W. Grota, "Snapseed Review: Best Photo-Editing App for Serious Photographers," tomsguide.com. Tom's Hardware, May 24, 2017. 